\title{
Estudio comparativo de técnicas de optimización multirespuesta en diseños experimentales
}

\section{Comparative study of multi-response optimization techniques in experimental designs}

\section{Barrios-Córdova Abel}

Colegio de Postgraduados, México

Departamento de estadística

Correo: barrios.abel@colpos.mx

https://orcid.org/0000-0001-9671-9463

Vaquera-Huerta Humberto

Colegio de Postgraduados, México

Departamento de estadística

Correo: hvaquera@colpos.mx

https://orcid.org/0000-0002-2805-804X

Romero-Padilla Juan Manuel

Colegio de Postgraduados, México

Departamento de estadística

Correo: romero.manuel@colpos.mx

https://orcid.org/0000-0002-2654-5266

\author{
Crossa Jose \\ Centro Internacional de Mejoramiento de Maíz y Trigo (CIMMYT), \\ México \\ Unidad de biometría y estadística \\ Correo: j.crossa@cgiar.org \\ https://orcid.org/0000-0001-9429-5855 \\ Burguete-Hernández Esteban \\ Colegio de Postgraduados, México \\ Departamento de estadística \\ Correo: burguete.esteban@colpos.mx \\ https://orcid.org/0000-0002-1450-3165
}

\section{Resumen}

En este trabajo se desarrolla una propuesta para comparar diferentes metodologías de optimización multirespuesta aplicadas a superficies de respuesta (RSM) en diseños experimentales, como herramientas de solución a problemas presentes principalmente en el área industrial. Se estudian las siguientes metodologías: función de deseabilidad (DES), MOORA (MOO), TOPSIS (TOP), MULTIMOORA (MMO), MOORA AD (MAD), TOPSIS AD (TAD) y redes neuronales multicapa (con los paquetes Neuralnet (NEU) y Nnet $(\mathrm{NET})$ ). Cada una de estas técnicas se aplican a tres casos de interés comercial o industrial con diferentes diseños experimentales (Taguchi, Box-Behnken y Diseño Central Compuesto), en un estudio de simulación Monte Carlo, donde se consideran como factores las diferentes técnicas comparadas, el tipo de diseño experimental y diferentes escenarios de correlaciones. Se comparan las técnicas por medio de una métrica que evalúa la distancia de cada respuesta estimada respecto a su valor ideal o deseado, con el fin de analizar las ventajas y desventajas de cada método. Los resultados obtenidos son consistentes en cada uno de los casos abordados y se concluye que las redes neuronales Neuralnet (NEU) son el mejor método; en segundo lugar, la función de deseabilidad (DES) y las redes neuronales Nnet (NET). Además, se encontró que el método MOORA AD (MAD) propuesto, tiene un excelente desempeño en un caso de estudio particular. Se recomienda en estudios comparativos futuros, emplear más tipos de diseños experimentales y aplicar más técnicas de optimización multirespuesta disponibles, con el fin de obtener mayor información sobre los escenarios y condiciones que muestren mejor desempeño así como realizar sugerencias de implementación más puntuales. Todo el desarrollo se realizó en R (R Core Team, 2019) con el fin de promover el uso de software libre con fines de investigación o desarrollo comercial. Descriptores: Optimización, optimización multiobjetivo, optimización multirespuesta, deseabilidad, optimización MCDM, redes neuronales artificiales, diseños experimentales.

\section{Abstract}

In this work a proposal is developed to compare different multi-response optimization methodologies applied to response surfaces (RSM) in experimental designs, as solution tools of problems mainly in the industrial area. The following methodologies are studied: desirability function (DES), MOORA (MOO), TOPSIS (TOP), MULTIMOORA (MMO), MOORA AD (MAD), TOPSIS AD (TAD) and multilayer neural networks (with Neuralnet (NEU) and Nnet (NET) packages). Each of these techniques is applied in three cases of commercial or industrial interest with different experimental designs (Taguchi, Box-Behnken and Central Composite Design), in a Monte Carlo simulation study, the techniques to be compared, the type of experimental design and different correlation scenarios are used as factors. The techniques are compared by a metric that evaluates the distance of each estimated response with respect to its ideal or desired value, in order to analyze the advantages and disadvantages of each method. The results obtained were consistent in each of the analyzed cases, it is concluded that Neuralnet Neural Networks (NEU) are the best method, secondly, the Desirability Function (DES) and Nnet Neural Networks (NET). In addition, the proposed MOORA AD (MAD) method was found to have excellent performance in one particular study case. It is recommended in future comparative studies, to use more types of experimental designs and to apply more multi-response optimization techniques available, in order to obtain more information on which scenarios and conditions the methods show a better performance and to make more specific implementation suggestions. All the programs were done using R (R Core Team, 2019) to promote the use of free software for research or commercial development purposes.

Keywords: Optimization, multiobjective optimization, multi-response optimization, desirability, MCDM optimization, artificial neural networks, experimental designs. 


\section{INTRODUCCIÓN}

La optimización de procesos frecuentemente se estudia con diseños de experimentos y métodos de superficies de respuesta. De acuerdo con Khuri (2017) la RSM incluye tres fases:

a) Selección y construcción de un diseño experimental apropiado que genere información adecuada y confiable de las variables respuesta $y$

b) Ajustar un modelo estadístico a partir del diseño experimental, que es una aproximación funcional de las relaciones entre las variables respuesta $y$ y un conjunto de variables de control (factores de estudio, $\left.x_{1}, x_{2}, \ldots, x_{k}\right)$

c) Obtener los niveles óptimos en las variables de control que producen los valores de respuesta máximas (o mínimas) en ciertas regiones de interés.

En muchas situaciones reales la optimización involucrará diversas variables, en este caso, el problema no es trivial. Comúnmente se usa el enfoque univariado de optimización, pero se tiene el problema de que no necesariamente se obtiene un óptimo común a todos los factores de estudio. A pesar de ello, la idea es buscar el mejor balance entre las variables involucradas buscando una solución de compromiso que permita optimizar el proceso y mejore la calidad del producto o servicio de interés (Gutiérrez et al., 2012).

En el caso multi respuesta en RSM, existen varias propuestas para resolver el problema de optimización. Khuri y Conlon (1981) desarrollaron un algoritmo para la optimización simultánea de varias funciones de respuesta que dependen del mismo conjunto de variables controlables y están adecuadamente representadas por modelos de regresión polinómica del mismo grado. Antony (2000) propone un enfoque alternativo utilizando el análisis de componentes principales en funciones de perdida de Taguchi. Otro enfoque es el uso de funciones de deseabilidad Akteke et al. (2015), las funciones de deseabilidad desempeñan un papel cada vez mayor para resolver la optimización. Para consultar otras metodologías usadas en optimización multiobjetivo ver Šibalija y Majstorović (2016).

El objetivo del presente trabajo fue comparar los siguientes métodos de optimización multiobjetivo: función de deseabilidad (DES), MOORA (MOO), TOPSIS (TOP), MULTIMOORA (MMO), MOORA AD (MAD), TOPSIS AD (TAD) y redes neuronales multicapa (con los paquetes Neuralnet (NEU) y Nnet (NET)), a partir de un experimento de simulación Monte Carlo considerando como factores de estudio los métodos menciona- dos anteriormente, el diseño experimental y la correlación entre las respuestas.

\section{Desarrollo}

La RSM sobre la región de interés implica la recopilación de datos experimentales, se debe elegir un modelo adecuado que se ajuste a esos datos y probar la idoneidad del modelo ajustado (Bayramov et al., 2004). Para superficies de respuesta de segundo orden típicamente se ajustan modelos que permitan estudiar la curvatura (efectos cuadráticos) y las interacciones entre los factores. El modelo de segundo orden es:

$y=\beta_{0}+\sum_{i=1}^{k} \beta_{i} x_{i}+\sum_{i=1}^{k} \sum_{j=1}^{k} \beta_{i j} x_{i} x_{j}+\sum_{i=1}^{k} \beta_{i j} x_{i}^{2}+\epsilon$

los diseños de segundo orden utilizados en este trabajo son el diseño central compuesto (DCC), Box-Behnken y diseño Taguchi. Se pueden ver aplicaciones de cada uno en Asadi y Zilouei (2016), Manohar et al. (2013), Amir et al. (2015), respectivamente. Los modelos ajustados obtenidos se emplean en el método DES, MAD y TAD, los demás métodos no necesitan ajustar un modelo.

\section{Métodos de OptIMIZACIÓN MULTIOBJetivo USADOS}

\section{FUNCIÓN DE DESEABILIDAD}

Es la técnica más utilizada en la industria actualmente, ya que se encuentra programada en diversos software comerciales, se propuso originalmente por Harrington (1965) proponiendo transformaciones exponenciales, posteriormente Derringer y Suich (1980) y Derringer (1994) mejoran la propuesta con funciones más flexibles; Akteke et al. (2015) muestran un ejemplo de aplicación. El método consiste en los siguientes pasos:

Paso 1. Teniendo los modelos ajustados se convierte cada respuesta $y_{i}$ en función de deseabilidad $d_{i}$, considerando si se desea maximizar, minimizar o lograr un valor objetivo.

Paso 2. Combinar las deseabilidades individuales por medio de la Deseabilidad Global, que es una media geométrica. Considerar un vector de pesos $w$ que pondera la importancia de cada respuesta.

$D G(x)=\left(\prod_{i=1}^{k} d_{i}^{w i}\right)^{\frac{1}{2 w_{i}}}$ 
Paso 3. Maximizar la deseabilidad global por medio de un algoritmo como Levenberg-Marquardt o NelderMead, considerando los límites de control para cada variable.

Paso 4. Localizar el vector óptimo global de las variables de control que se usa para poder predecir las respuestas óptimas deseadas.

\section{MOORA}

Los métodos de toma de decisiones con criterios múltiples (MCDM), generan como resultado un índice o ranking de las mejores respuestas encontradas (Miettinen, 2008).

El método MOORA (Multi-Objective Optimization on the Basis of Ratio Analysis) propuesto por Brauers y Zavadskas (2006) realiza las sumas de rendimientos normalizados de costo beneficio y calcula su diferencia para representar el rendimiento global de cada una de las alternativas en forma de un índice o ranking. Los pasos son los siguientes:

Paso 1. Se identifican las variables independientes $x_{i j}, i=1,2, \ldots, n ; j=1,2, \ldots, r$ donde $n$ es el número de situaciones experimentales y $r$ son el número de respuestas. Se construye la matriz de decisión.

$D=\left[\begin{array}{cccc}x_{11} & x_{12} & \cdots & x_{1 r} \\ x_{21} & x_{22} & \cdots & x_{2 r} \\ \vdots & \vdots & \vdots & \vdots \\ x_{n 1} & x_{n 2} & \cdots & x_{n r}\end{array}\right]$

Paso 2. Normalizar la matriz de decisión, se divide cada valor de $x_{i j}$ entre su norma euclidiana para normalizar cada elemento $x_{i j}^{*}$ y obtener la matriz normalizada $D^{*}$ (Yang \& Chou, 2005):

$x_{i j}^{*}=\frac{x_{i j}}{\sqrt{\sum_{i=1}^{n} x_{i j}^{2}}}, \quad i=1,2, \ldots, n ; \quad j=1,2, \ldots, r$

Paso 3. Se obtiene la matriz de decisión normalizada ponderada $V$ que se calcula multiplicando cada elemento de la matriz $D^{*}$ por el peso correspondiente a la importancia de cada respuesta $w_{j}$ y que cumple $\sum_{j=1}^{r} w_{j}=1$ :

$V=\left[X_{i, j}\right]_{m \times r}, \quad i=1,2, \ldots, n ; \quad j=1,2, \ldots, r$

$X_{i j}=x_{i j}^{*} w_{j}$
Paso 4. Calcular el índice MOORA $Y_{i}$ :

$Y_{i}=\sum_{j=1}^{t} X_{i j}-\sum_{j=t+1}^{r} X_{i j}$

Donde:

$t=$ número de respuestas a maximizar

$(r-t) \quad=$ número de respuestas a minimizar

$Y_{i} \quad=$ ranking del $i$-ésimo caso experimental con respecto a todas las respuestas.

A mayor valor de $Y_{i}$ se produce un mejor rendimiento de respuesta múltiple.

\section{MOORA AD}

En el presente trabajo se propone el uso de una propiedad aditiva que aparece en Phadke (1989) en diseño robusto, y se utiliza en el método MOORA en Kuo et al. (2008) para casos de optimización empleando técnicas multicriterio, considerando la selección de factores se propone un método denominado MOORA AD añadiendo un paso 5 en el método MOORA.

Paso 5. Calcular un promedio del efecto de cada factor en sus diferentes niveles y elegir el mayor de ellos como mejor respuesta.

\section{TOPSIS}

El método TOPSIS (Technique for Order of Preference by Similarity to Ideal Solution) desarrollado por Hwang y Yoon (1981), es un ranking basado en dimensiones que elige la alternativa de que simultáneamente tiene la distancia más corta de la solución ideal positiva (maximiza beneficios y minimiza el costo) y la distancia más alejada de la solución ideal negativa (maximiza el costo y minimizan los beneficios) (Majid et al., 2012). Los pasos son los siguientes:

Paso 1 a 3. Idénticos al método MOORA (MOO).

Paso 4. Calcular la solución ideal positiva y negativa:

$$
\begin{aligned}
& d^{+}=\left(X_{1}^{+}, X_{2}^{+}, \ldots, X_{r}^{+}\right) \\
& X_{j}^{+}=\left\{\left(\max _{i} X_{i j} \mid j \in J\right), i=1,2, \ldots, n\right\} \\
& d^{-}=\left(X_{1}^{-}, X_{2}^{-}, \ldots, X_{r}^{-}\right)
\end{aligned}
$$


$X_{j}^{-}=\left\{\left(\min _{i} X_{i j} \mid j \in J^{\prime}\right), i=1,2, \ldots, n\right\}$

donde J está relacionado con algún criterio de beneficio y J'está relacionado con algún criterio de costo.

Paso 5. Calcular la distancia del $i$-ésimo experimento a la solución ideal positiva y negativa:

$$
\begin{aligned}
& d_{i}^{+}=\sqrt{\sum_{j=1}^{r}\left(X_{i j}-X_{j}^{+}\right)^{2}}, \quad i=1,2, \ldots, n ; \quad j=1,2, \ldots, r \\
& d_{i}^{-}=\sqrt{\sum_{j=1}^{r}\left(X_{i j}-X_{j}^{-}\right)^{2}}, \quad i=1,2, \ldots, n ; \quad j=1,2, \ldots, r
\end{aligned}
$$

Paso 6. Calcular el índice TOPSIS $C_{i}$, también llamado coeficiente de cercanía:

$C_{i}=\frac{d_{i}^{-}}{d_{i}^{-}+d_{i}^{+}}, \quad i=1,2, \ldots, n ; \quad j=1,2, \ldots, r$

\section{TOPSIS AD}

Así como en el método MOORA, se propone utilizar la propiedad aditiva propuesta por Phadke (1989). El método TOPSIS AD surge añadiendo esta propiedad como paso 7 en el método TOPSIS.

Paso 7. Calcular un promedio del efecto de cada factor en sus diferentes niveles y elegir el mayor de ellos como mejor respuesta.

\section{MULTIMOORA}

El método MOORA puede tener una variante en el paso 4, en lugar del índice "ratio system", se realiza un "Reference point", y la combinación de estos índices junto con el índice "Full multiplicative form" genera el índice MULTIMOORA propuesto por Brauers y Zavadskas (2010). El Ratio system emplea la sumatoria como operador de agregación con los datos normalizados, el Reference point calcula un punto ideal de referencia y mide las distancias de las alternativas a este punto ideal. El Full multiplicative form usa como operador de agregación la multiplicación; se calcula el ranking de cada índice y finalmente se calcula el ranking MULTIMOORA por medio de la teoría de dominancia. La metodología se menciona en Ceballos et al. (2015) y los pasos son los siguientes:

Paso 1 a 4. Son idénticos al método MOORA (MOO).

Paso 5. Cálculo del índice de Reference point $P_{i}$, primero se calcula el punto de referencia como:

$R F=\left\{v_{1}^{+}, v_{2}^{+}, \ldots, v_{n}^{+}\right\}$ donde $v_{j}^{+}=\max _{i}\left(v_{i j}\right)$ si el $j$-ésimo criterio es de beneficio, y $v_{j}^{-}=\min _{i}\left(v_{i j}\right)$ si es de coste. Posteriormente se aplica la métrica min-max de Chebyshev:

$P_{i}=\underset{\min }{i}\left(j_{\max }\left|v_{j}^{+}-v_{i j}\right|\right)$

las alternativas se ordenan de forma creciente y las mejores son las de menor valor.

Paso 6. Cálculo de la ecuación completa multiplicativa, $U_{i}$ (Full multiplicative form):

$U_{i}=\frac{\prod_{j=1}^{t} x_{i j}^{w_{j}}}{\prod_{j=t+1}^{r} x_{i j}^{w_{j}}}$

donde el numerador son los criterios de beneficio y el denominador son los de coste. Después las alternativas se ordenan de forma decreciente, siendo las mejores alternativas las de valor mayor.

Paso 7. Teoría de dominancia (Brauers et al., 2011; Brauers y Zavadskas, 2011). Se unen los rankings por medio de la teoría de dominancia y se genera un ranking final. Se considera que los tres índices tienen la misma importancia.

\section{Redes Neuronales Artificiales (ANN)}

Las Redes Neuronales Artificiales (ANN) son modelos computacionales inspirados en el sistema nervioso de los seres vivos, con capacidad de adquirir y mantener conocimientos (basados en información) y pueden definirse como un conjunto de unidades de procesamiento, representadas por neuronas artificiales, interconectadas por muchas interconexiones (sinapsis artificiales), implementadas por vectores y matrices de pesos sinápticos (Da Silva et al., 2017).

En este trabajo se usaron redes multicapa, donde el número de neuronas de la capa de entrada coincide con las variables de entrada. En la capa oculta el número de neuronas dependerá de la complejidad del problema y de la cantidad de información alimentada. La cantidad de neuronas de salida depende de las señales de respuesta estudiadas. Se emplean redes con función de activación sigmoidea y algoritmo de entrenamiento backpropagation, en el caso del método NEU se usa resilient backpropagation con retroceso de pesos $($ rprop +$)$ y para el método NET se usa backpropagation con optimización BFGS. Se pueden ver otros métodos de entrenamiento y algoritmos de optimización en Berzal (2018). 
Se han empleado redes neuronales artificiales en diversos trabajos de optimización que se pueden observar en Maran y Priya (2015), Kilickap et al. (2017), Najafi et al. (2018), Shafi et al. (2018), Kim et al. (2019), entre otros.

La metodología del uso de redes neuronales multicapa en optimización multiobjetivo de Tong y Hsieh (2001), propone:

Paso 1. (Red inversa). Entrenar una red neuronal con las respuestas observadas como entradas y los factores de control como salidas.

Paso 2. Obtenida la red entrenada se predice con los datos de las respuestas deseadas para obtener los factores de entrada optimizados.

Paso 3. (Red directa). Entrenar una red neuronal con los factores de control como entradas y las respuestas observadas como salidas.

Paso 4. Obtenida la red entrenada se realiza la predicción con los factores de entrada optimizados del paso 2 y así obtener los valores objetivos optimizados.

\section{Simulación Monte Carlo}

Se realizó un estudio de simulación para analizar la efectividad de los métodos, el experimento Monte Carlo consistió en lo siguiente:

Factores de estudio:

- Factor método: Se emplearon ocho métodos de estudio a comparar, mencionados en la sección previa.

- Factor tipo de Diseño Experimental: Se usan tres situaciones con diferentes diseños experimentales:

- Diseño Taguchi (L8): con cuatro factores de control y dos respuestas.

- Diseño Box-Behnken: con tres factores de control y dos respuestas.

- Diseño Central Compuesto: con tres factores de control y seis respuestas.

- Factor correlación en superficie de respuesta con 7 niveles de correlación $(0.9,0.5,0.2,0,-0.2,-0.5$, - 0.9). Que indican correlación entre respuestas alta, media y baja, tanto positiva como negativa, y considera al centro el caso independiente.

De los anteriores factores se obtuvieron 168 combinaciones que se estudiaron con 5000 réplicas por cada experimento, obteniendo como respuesta un vector de respuestas medias y un vector de varianzas. $\mathrm{Al}$ vector de respuestas medias se le aplicó una métrica para poder comparar la efectividad de cada método.

\section{Métrica usada para comparar los métOdos}

Se emplearon dos métricas:

La primera es el GPE, Error Porcentual Global (Global Percentage Error), que evalúa la distancia de la respuesta estimada respecto a su valor ideal o deseado, el mejor valor de esta métrica es el cero o muy cercano a este (Rocha et al., 2015), su expresión en la siguiente:

$G P E=\sum_{i=1}^{m}\left|\frac{\hat{y}_{i}}{T_{i}}-1\right|$

Donde:

$\hat{y}_{i}=$ respuesta media estimada

$T_{i}=$ valor objetivo desado $\mathrm{y}$

$m$ = cantidad de respuestas

La segunda métrica aparece en Costa y Lourenço (2016) llamada desviación relativa del objetivo, RTD (Relative Target Deviation), que mide la distancia de la respuesta estimada respecto al valor deseado, es adimensional y entre más bajo el valor mejor, cero es el valor ideal, que indica que todas las respuestas están en el objetivo. Su expresión es:

$R T D=\sum_{i=1}^{m} \frac{\left|\hat{y}_{i}-T_{i}\right|}{T_{i}}$

En el cálculo de ambas métricas se obtienen los mismos resultados, por lo que se reportan los resultados como GPE/RTD.

\section{IMPLEMENTACIÓN EN SOFTWARE R}

Todos los métodos y simulación fueron programados en software R (R Core Team, 2019). En la Figura 1 se ilustra el método usado, el cual consistió en los siguientes pasos:

1. Programar la función de deseabilidad con el paquete desirability (Kuhn, 2016).

2. Programar los métodos multicriterio MOORA, TOPSIS, MMOORA, MOORA AD y TOPSIS AD, con ayuda del paquete MCDM (Ceballos, 2016).

3. Programar las redes neuronales con la idea de Tong y Hsieh (2001), para el método NEU se usó el paquete Neuralnet (Fritsch et al., 2019), para el método NET se empleó el paquete Nnet (Venables \& Ripley, 
2016). La cantidad de neuronas por cada capa oculta se obtuvo por prueba y error con pruebas de 3 a 12 neuronas (esto para todos los casos). Obteniendo las siguientes arquitecturas: Caso 1: NEU, red inversa y directa $(2,10,8,4)$ y $(4,10,8,2)$, respectivamente; NET, red inversa y directa $(2,3,4)$ y $(4,4,2)$. Caso 2 : NEU, red inversa y directa $(2,10,8,3)$ y $(3,10,8,2)$; NET, red inversa y directa $(2,3,3)$ y $(3,4,2)$. Caso 3 : NEU, red inversa y directa $(6,3,3)$ y $(3,4,6)$; NET, red inversa y directa $(6,3,3)$ y $(3,3,6)$.

4. Realizar una regresión para generar los modelos que ajustan a cada una de las respuestas.

5. Con ayuda del paquete mvtnorm (Genz et al., 2019), se generan normales multivariadas con media $=0$, varianza $=1$ y con una matriz de correlación (donde se controla la correlación entre las respuestas). Estas normales multivariadas se agregan en forma de residuales a los modelos ajustados, y sobre estos nuevos modelos se implementan cada una de las metodologías. Se realizaron 5000 repeticiones en esta simulación obteniendo un vector de medias y un vector de varianzas para cada respuesta y para cada método.

6. Aplicar la métrica GPE/RTD en cada uno de los métodos.

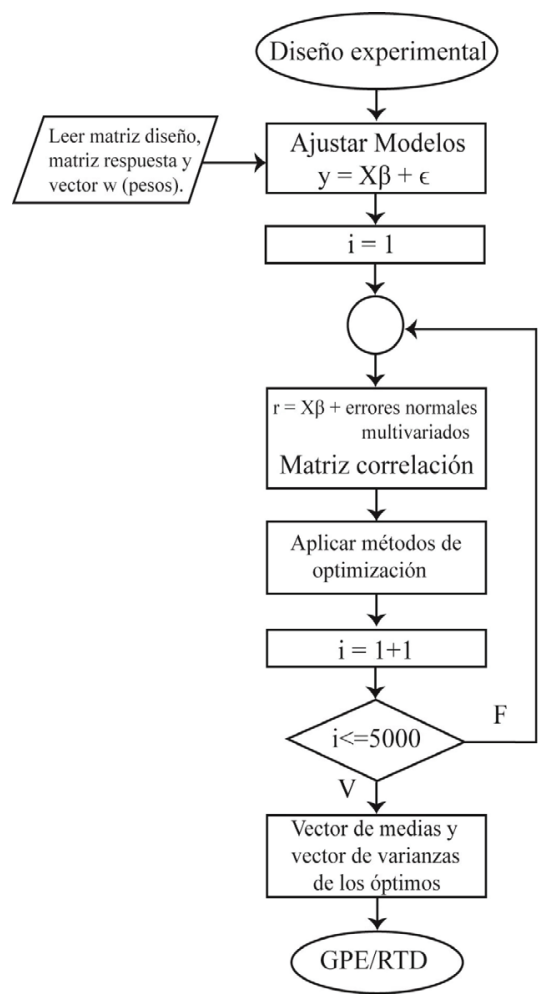

Figura 1. Metodología propuesta en la comparación de los métodos multirespuesta estudiados

\section{DisCUSIÓN Y ANÁLISIS DE RESULTADOS}

\section{Caso 1: Diseño Taguchi}

Este caso aparece en Ic \& Yildirim (2012) y en Ic \& Yildirim (2013). Se desea mejorar la calidad de un modelo de lavadora para un fabricante de lavadoras en Turquía. Donde se emplea el diseño Taguchi , que considera cuatro factores controlables con dos niveles cada uno, los cuales caracterizan la calidad de la lavadora: $x_{1}$ profundidad del panel lateral del cuerpo de la lavadora, $x_{2}$ grosor del panel lateral del cuerpo de la lavadora, $x_{3}$ tipo de aislamiento del motor de la lavadora y $x_{4}$ grosor de la correa del motor de la lavadora. Las dos respuestas deseadas son: $y_{1}$ nivel de ruido de la lavadora $(\mathrm{dB})$ (minimizar) y velocidad de giro (rpm) (maximizar). El diseño experimental se puede observar en la Tabla 2 en Ic \& Yildirim (2013). Además, se consideran 0.7 y 0.3 como pesos de cada respuesta, ya que es más importante la disminución del ruido. Note que las respuestas son transformadas a señal ruido Taguchi (SN) dependiendo de la necesidad de maximizar, minimizar o lograr un target. El óptimo reportado es igual al deseado: $y_{1}=-34.15(\mathrm{SN})=51 \mathrm{~dB}, y_{2}=64.51(\mathrm{SN})=1680 \mathrm{rpm}$. Los modelos usados se muestran en la Tabla 1.

La Tabla 2 muestra la varianza en la obtención de los óptimos para cada método, y la Tabla 3 contiene los valores de GPE/RTD. 
Tabla 1. Modelos ajustados para el Caso 1

\begin{tabular}{ccc}
\hline Modelo de regresión & $\mathrm{R}^{2}$ aj. & p-valor \\
\hline$S N_{1}=37.21-0.77 x_{1}+1.12 x_{2}+0.52 x_{3}+0.53 x_{4}-0.31 x_{3} x_{4}$ & 0.9974 & 0.0018 \\
$S N_{2}=61.18+0.77 x_{1}+1.52 x_{2}-0.86 x_{3-}-0.46 x_{4}-0.20 x_{2} x_{4}+0.47 x_{3} x_{4}$ & 0.9998 & 0.0095 \\
\hline
\end{tabular}

Tabla 2. Varianzas por método para el Caso 1

\begin{tabular}{ccccccccc}
\hline Correlación & DES & MOO & TOP & MAD & TAD & MMO & NEU & NET \\
\hline 0.9 & 0.853 & 1.877 & 1.819 & 1.860 & 1.927 & 1.740 & 0.951 & 1.298 \\
0.5 & 1.070 & 1.895 & 1.823 & 1.930 & 1.969 & 1.790 & 0.825 & 1.323 \\
0.2 & 1.222 & 1.822 & 1.779 & 1.989 & 2.045 & 1.730 & 0.746 & 1.058 \\
0.0 & 1.196 & 1.696 & 1.672 & 1.883 & 1.916 & 1.640 & 0.598 & 0.985 \\
-0.2 & 1.238 & 1.611 & 1.591 & 1.878 & 1.907 & 1.571 & 0.535 & 0.864 \\
-0.5 & 1.192 & 1.411 & 1.403 & 1.805 & 1.840 & 1.389 & 0.341 & 0.537 \\
-0.9 & 1.042 & 1.085 & 1.086 & 1.665 & 1.680 & 1.084 & 0.086 & 0.212 \\
\hline
\end{tabular}

Tabla 3. GPE/RTD por método para el Caso 1

\begin{tabular}{ccccccccc}
\hline Correlación & DES & MOO & TOP & MAD & TAD & MMO & NEU & NET \\
\hline 0.9 & 0.019 & 0.032 & 0.032 & 0.025 & 0.026 & 0.030 & 0.019 & 0.022 \\
0.5 & 0.017 & 0.028 & 0.029 & 0.021 & 0.023 & 0.026 & 0.016 & 0.020 \\
0.2 & 0.017 & 0.024 & 0.024 & 0.018 & 0.020 & 0.022 & 0.014 & 0.017 \\
0 & 0.020 & 0.021 & 0.022 & 0.016 & 0.017 & 0.020 & 0.011 & 0.015 \\
-0.2 & 0.024 & 0.020 & 0.020 & 0.015 & 0.016 & 0.019 & 0.009 & 0.013 \\
-0.5 & 0.028 & 0.023 & 0.023 & 0.018 & 0.017 & 0.023 & 0.006 & 0.010 \\
-0.9 & 0.033 & 0.027 & 0.027 & 0.021 & 0.021 & 0.027 & 0.002 & 0.005 \\
\hline
\end{tabular}

La varianza de la estimación NEU y NET muestran los valores más bajos seguidos de DES, MMO, MOO y TOP; y los valores más altos se observan en MOA y TOA (Tabla 2). Considerando el desempeño a diferentes correlaciones, se tiene que al aumentar positivamente la correlación los valores del GPE/RTD aumentan gradualmente para todas las metodologías, excepto para DES donde se observa una ligera disminución; en el caso de aumentar negativamente la correlación los valores del GPE/RTD tienen un aumento gradual en todos los métodos, excepto para ambas redes neuronales donde se observa una disminución del error (Tabla 3).

El mejor método para este caso con diseño Taguchi con dos respuestas, son las redes neuronales porque presentan el menor valor de GPE/RTD (Tabla 3 y Figura 2).

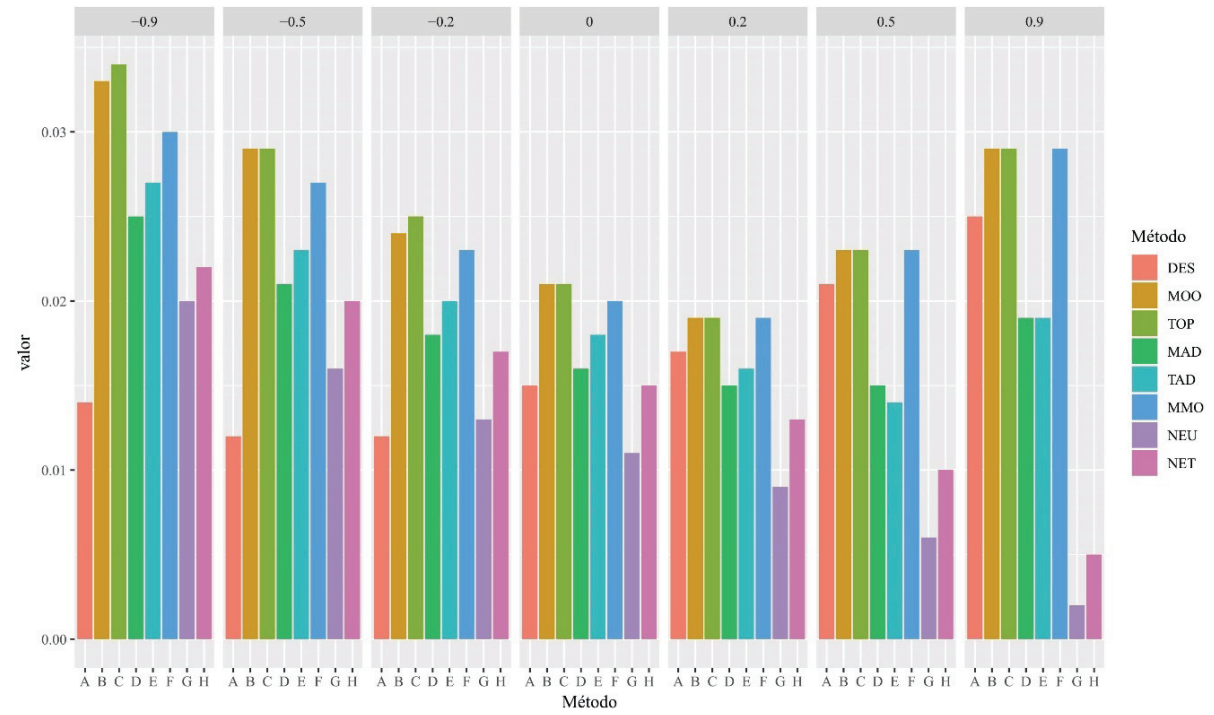

Figura 2. Métrica GPE/RTD para el Caso 1 


\section{CAso 2: Diseño Box-BehnKen}

Este caso se muestra en Lu et al. (2013), donde se estudia la extracción de carotenoides y clorofila del alga Laminaria japonica Aresch, que es un alga de interés comercial. Se usa un diseño Box-Behnken y los factores de control son: $x_{1}$ temperatura de extracción $(\mathrm{K}), x_{2}$ presión de extracción (MPa) y $x_{3}$ cantidad de cosolvente (\%). Las respuestas son: $y_{1} \mathrm{~g}$ de carotenoides $\left(\mathrm{kg}^{-1}\right.$ masa seca de biomasa) (max) y $y_{2}$ g de clorofila $\left(\mathrm{kg}^{-1}\right.$ masa seca de biomasa) (max). El diseño experimental se muestra en la Tabla 2 en Lu et al. (2013). Se consideran 0.5 y 0.5 como pesos de cada respuesta. El óptimo deseado es: $y_{1}=0.251$, $y_{2}=2.473$. El óptimo reportado obtenido de un experimento comprobatorio es: $y_{1}=0.233, y_{2}=2.335$. La Tabla 4 exhibe los modelos cuadráticos usados.

La Tabla 5 exhibe las varianzas en la obtención de los óptimos para cada método.
En la Tabla 6 se observan los valores de GPE/RTD para el caso 2.

De acuerdo con la Tabla 5 podemos indicar que la varianza de las redes neuronales es la más baja respecto a las demás. Al aumentar positivamente la correlación los valores del GPE/RTD tienen un aumento gradual para todas las metodologías, excepto para las redes neuronales donde se observa una ligera disminución del error; en caso de aumentar negativamente la correlación los valores del GPE/RTD tienen un aumento gradual en todos los métodos, excepto para la DES que presenta una disminución del error (Tabla 6).

Para el caso con diseño Box-Behnken con dos respuestas, las redes neuronales tienen el mejor desempeño al presentar los valores más bajos de GPE/RTD (Tabla 6 y Figura 3).

Tabla 4. Modelos ajustados para el Caso 2

\begin{tabular}{ccc}
\hline Modelo de regresión & $\mathrm{R}^{2}$ aj. & $\mathrm{p}$-valor \\
\hline$y_{1}=0.2302+0.0404 x_{1}+0.0334 x_{2}-0.0375 x_{3}-0.0828 x_{1}{ }^{2}-0.0475 x_{3}{ }^{2}$ & 0.8643 & 0.0085 \\
$y_{2}=2.04+.27 x_{1}+.18 x_{2}+.11 x_{3}-.31 x_{1}{ }^{2}-.09 x_{2}{ }^{2}+.07 x_{3}{ }^{2}+.09 x_{1} x_{2}+.09 x_{1} x_{3}+.08 x_{2} x_{3}$ & 0.9278 & 0.0019 \\
\hline
\end{tabular}

Tabla 5. Varianzas por método para el Caso 2

\begin{tabular}{ccccccccc}
\hline Correlación & DES & MOO & TOP & MAD & TAD & MMO & NEU & NET \\
\hline 0.9 & 0.809 & 0.702 & 0.700 & 1.169 & 1.194 & 0.746 & 0.081 & 0.070 \\
0.5 & 0.830 & 1.058 & 0.933 & 1.281 & 1.289 & 0.999 & 0.150 & 0.072 \\
0.2 & 0.851 & 1.193 & 1.143 & 1.372 & 1.405 & 1.066 & 0.218 & 0.073 \\
0 & 0.804 & 1.274 & 1.193 & 1.368 & 1.403 & 1.077 & 0.240 & 0.075 \\
-0.2 & 0.750 & 1.375 & 1.261 & 1.400 & 1.452 & 1.072 & 0.274 & 0.075 \\
-0.5 & 0.649 & 1.393 & 1.231 & 1.376 & 1.386 & 0.927 & 0.327 & 0.074 \\
-0.9 & 0.411 & 1.103 & 1.093 & 1.252 & 1.218 & 0.508 & 0.373 & 0.077 \\
\hline
\end{tabular}

Tabla 6. GPE/RTD por método para el Caso 2

\begin{tabular}{ccccccccc}
\hline Correlación & DES & MOO & TOP & MAD & TAD & MMO & NEU & NET \\
\hline 0.9 & 6.425 & 6.894 & 6.895 & 4.371 & 4.413 & 6.909 & 0.247 & 0.430 \\
0.5 & 5.607 & 6.424 & 6.431 & 4.103 & 4.190 & 6.514 & 0.323 & 0.470 \\
0.2 & 4.892 & 6.157 & 6.153 & 3.905 & 3.977 & 6.056 & 0.385 & 0.501 \\
0 & 4.430 & 5.928 & 5.939 & 3.682 & 3.915 & 5.777 & 0.426 & 0.519 \\
-0.2 & 3.900 & 5.785 & 5.828 & 3.760 & 4.017 & 5.466 & 0.480 & 0.539 \\
-0.5 & 2.986 & 5.880 & 5.861 & 3.776 & 4.073 & 5.130 & 0.550 & 0.575 \\
-0.9 & 1.356 & 6.601 & 6.227 & 4.197 & 4.419 & 4.462 & 0.636 & 0.629 \\
\hline
\end{tabular}




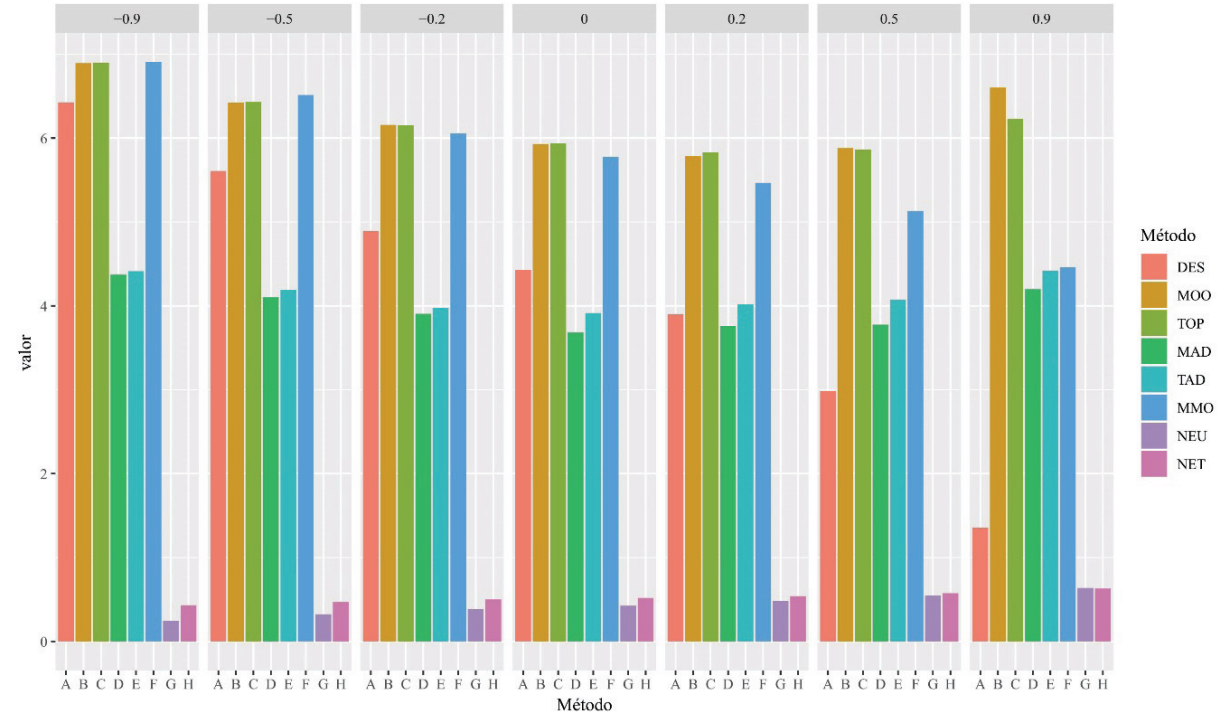

Figura 3. Métrica GPE/RTD para el Caso 2

\section{Caso 3: Diseño central compuesto}

Esta situación aparece en Sivakumar et al. (2007), donde se quiere desarrollar un método de HPLC de fase inversa para la determinación simultánea de domperidona (DP) y pantoprazol (PP) en dos preparaciones farmacéuticas comerciales. Se usa un diseño Central Compuesto con 14 experimentos por duplicado y 6 experimentos con puntos centrales, los factores de control son: $x_{1}$ composición de la fase móvil $(\%, \mathrm{v} / \mathrm{v}), x_{2}$ molaridad del buffer $(\mathrm{mM})$ y $x_{3}$ velocidad de flujo $(\mathrm{ml} /$ $\mathrm{min})$, con cinco niveles. Respuestas: $y_{1}$ factor de retención de PP, $y_{2}$ tiempo de retención de PP, $y_{3}$ tiempo de retención de IS (Acetofenona), $y_{4}$ tiempo de retención de DP, $y_{5}$ resolución entre PP-IS, $y_{6}$ resolución entre IS-DP. Para observar el diseño experimental empleado ver la Tabla 1 en Sivakumar et al. (2007). Ponderación (2, 1, 1, $4,2,1)$ para cada respuesta. El óptimo deseado es: $y_{1}=$ $1.25, y_{2}=3.26, y_{3}=3.72, y_{4}=5.92, y_{5}=2.00$ y $y_{6}=8.77$. El óptimo reportado es: $y_{1}=1.59, y_{2}=3.27, y_{3}=3.72, y_{4}=5.97$, $y_{5}=2.54$ y $y_{6}=10.40$. La Tabla 7 contiene los seis modelos cuadráticos usados.

La Tabla 8 muestra las varianzas para cada método para el caso 3. La Tabla 9 contiene los valores de GPE/ $R T D$ conseguidos de la simulación.

Tabla 7. Modelos ajustados para el Caso 3

\begin{tabular}{lcc}
\hline \multicolumn{1}{c}{ Modelo de regresión } & $\mathrm{R}^{2}$ aj. & $\mathrm{p}$-valor \\
\hline$y_{1}=1.738-0.3238 x_{1}+0.0327 x_{2}+0.0299 x_{1}^{2}-0.0131 x_{2} x_{3}$ & 0.9942 & $<2.2 \mathrm{e}-16$ \\
$y_{2}=4.1-0.49 x_{1}+0.05 x_{2}-0.5 x_{3}+0.04 x_{1}^{2}+0.07 x_{3}{ }^{2}+0.05 x_{1} x_{3}-0.03 x_{2} x_{3}$ & 0.9972 & $<2.2 \mathrm{e}-16$ \\
$y_{3}=4.57-0.33 x_{1}-0.57 x_{3}+0.09 x_{3}{ }^{2}$ & 0.9724 & $<2.2 \mathrm{e}-16$ \\
$y_{4}=8.11-1.66 x_{1}-0.20 x_{2}-1.00 x_{3}+0.33 x_{1}{ }^{2}+0.18 x_{3}{ }^{2}+0.20 x_{1} x_{2}$ & 0.975 & $<2.2 \mathrm{e}-16$ \\
$y_{5}=2.38+0.95 x_{1}-0.22 x_{2}$ & 0.8221 & $9.08 \mathrm{e}-13$ \\
$y_{6}=13.66-2.80 x_{1}-0.58 x_{2}-0.40 x_{3}$ & 0.9069 & $3.52 \mathrm{e}-16$ \\
\hline
\end{tabular}

Tabla 8. Varianzas por método para el Caso 3

\begin{tabular}{ccccccccc}
\hline Correlación & DES & MOO & TOP & MAD & TAD & MMO & NEU & NET \\
\hline 0.9 & 5.065 & 9.607 & 10.360 & 7.376 & 11.320 & 9.268 & 2.453 & 2.080 \\
0.5 & 4.646 & 10.906 & 11.749 & 6.241 & 11.286 & 11.184 & 2.608 & 2.237 \\
0.2 & 4.271 & 11.405 & 12.919 & 4.770 & 11.021 & 12.196 & 2.588 & 2.138 \\
0 & 0.536 & 11.746 & 13.111 & 2.964 & 10.505 & 12.889 & 2.469 & 1.946 \\
-0.2 & 0.530 & 10.333 & 13.026 & 1.161 & 9.603 & 12.684 & 2.257 & 1.566 \\
-0.5 & 0.564 & 12.026 & 14.604 & 1.553 & 10.221 & 13.934 & 2.419 & 1.587 \\
-0.9 & 0.576 & 14.662 & 16.151 & 2.046 & 11.005 & 15.860 & 2.524 & 1.651 \\
\hline
\end{tabular}


Tabla 9. GPE/RTD por método para el Caso 3

\begin{tabular}{ccccccccc}
\hline Correlación & DES & MOO & TOP & MAD & TAD & MMO & NEU & NET \\
\hline 0.9 & 1.320 & 2.902 & 3.038 & 0.914 & 1.487 & 2.863 & 1.314 & 1.442 \\
0.5 & 1.158 & 2.243 & 2.497 & 0.939 & 1.564 & 2.251 & 1.167 & 1.416 \\
0.2 & 1.063 & 1.719 & 2.033 & 0.944 & 1.694 & 1.763 & 0.998 & 1.394 \\
0 & 0.924 & 1.264 & 1.718 & 0.924 & 1.743 & 1.352 & 0.937 & 1.396 \\
-0.2 & 0.937 & 0.787 & 2.199 & 0.887 & 1.947 & 1.238 & 0.895 & 1.341 \\
-0.5 & 0.975 & 0.939 & 2.236 & 0.892 & 1.884 & 1.371 & 0.884 & 1.363 \\
-0.9 & 1.024 & 1.131 & 2.301 & 0.899 & 1.863 & 1.501 & 0.856 & 1.335 \\
\hline
\end{tabular}

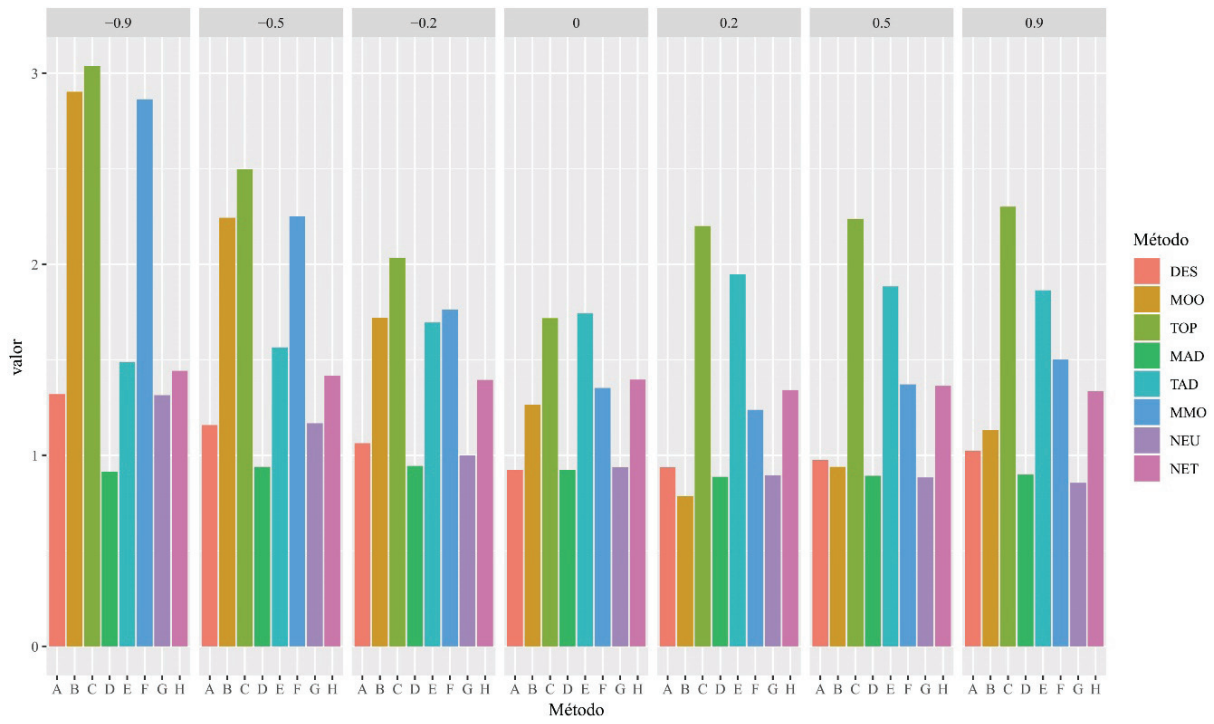

Figura 4. Métrica GPE/RTD para el Caso 3
En la varianza podemos ver que los métodos NEU y NET tienen las varianzas más bajas y el resto de los métodos tienen alta variabilidad en la estimación (Tabla 8).

En la Tabla 9 se observa que los métodos con los errores más pequeños son MAD y NEU, seguidos de DES y NET. Al aumentar positivamente la correlación los valores del GPE/RTD tienen un aumento gradual en las metodologías, excepto para MAD y TAD donde se observa una ligera disminución del error; en el caso de aumentar negativamente la correlación los valores del GPE/RTD tienen un aumento gradual en todos los métodos, excepto para NEU y NET que sufren una disminución del error.

En este caso las mejores metodologías son MAD y NEU, pero esta última tiene varianza más baja; después siguen los métodos DES y NET (Tabla 9 y Figura 4).

\section{CONCLUSIONES}

Considerando la métrica usada en el presente trabajo podemos concluir que el mejor método considerando los casos abordados y las condiciones mencionadas durante el desarrollo del experimento de simulación son las redes neuronales artificiales, particularmente el método NEU (Neuralnet) de redes multicapa al obtener los valores de GPE/RTD más pequeños. En segundo lugar, se pueden mencionar a las redes neuronales multicapa de tipo NET (Nnet) y la función de Deseabilidad (DES) que logran un buen desempeño, pero al aumentar las respuestas ambos métodos empiezan a sufrir de mayores errores, e incluso la función de Deseabilidad puede encontrar respuestas fuera de rango. Las técnicas MCDM no presentaron un buen desempeño, ya que no son capaces de encontrar valores intermedios en los 
factores de control; pero es importante resaltar que el método MOORA AD (MAD) logra un excelente resultado equiparable con el método NEU en casos donde se incrementan sustancialmente las respuestas, por lo que se recomienda su aplicación en este tipo de situaciones.

\section{AgradeCimientos}

El presente artículo es producto de la tesis de maestría que el Consejo Nacional de Ciencia y Tecnología tuvo a bien auspiciar económicamente en su totalidad. Muchas gracias.

\section{RefERENCIAS}

Akteke-Öztürk, B., Weber, G.-W. \& Köksal, G. (2015). Desirability functions in multiresponse optimization. En Plakhov A.T. (Ed.), Optimization in the Natural Sciences 129-146. Cham.: Springer International Publishing.

Amir, E., Milad, M., Soheil, A.N. \& Mohammad, M.S. (2015). Investigation of aluminum. Energy Technology \& Policy, 2(2), 19-27.

Antony, J. (2000). Multi-response optimization in industrial experiments using taguchi's quality loss function and principal component analysis. Quality and Reliability Engineering International, 16(1), 3-8. https://doi.org/10.1002/(SICI)1099-1638(200001/02)16:1\%3C3::AIDQRE276\%3E3.0.CO;2-W

Asadi, N. \& Zilouei, H. (2016). Optimization of organosolv pretreatment of rice straw for enhanced biohydrogen production using enterobacter aerogenes. Bioresource Technology, 227, 335344. http://doi.org/10.1016/j.biortech.2016.12.073

Bayramov, F., Taşdemir, C. \& Taşdemir, M.A. (2004). Optimisation of steel by means of statistical response surface method. Cement and Concrete Composites, 26(6), 665-675.

Berzal, F. (2018). Redes Neuronales \& Deep Learning. Granada, España: Independently published.

Brauers, W.K. \& Zavadskas, E.K. (2006). The moora method and its application to privatization in a transition economy. Control and Cybernetics, 35(2), 445-469.

Brauers, W.K. \& Zavadskas, E.K. (2010). Project management by multimoora as an instrument for transition economies. Technological and Economic Development of Economy, 16, 5-24.

Brauers, W. \& Zavadskas, E. (2011). Multimoora optimization used to decide on a bank loan to buy property. Technological and Economic Development of Economy, 17, 174-188. https://doi. org/10.3846/13928619.2011.560632

Brauers, W., Balezentis, A. \& Balezentis, T. (2011). Multimoora for the eu member states updated with fuzzy number theory. Technological and Economic Development of Economy, 17, 259-290. Recuperado de https://www.researchgate.net/deref/http $\% 3 \mathrm{~A} \% 2 \mathrm{~F} \% 2 \mathrm{Fdx}$. doi.org\%2F10.3846\%2F20294913.2011.580566

Ceballos, B.A. (2016). MCDM: Multi-Criteria Decision Making Methods for Crisp Data. R package version 1.2.
Ceballos, B., Lamata, M.T. \& Pelta, D. (2015). Una comparativa de modelos de decisión multi-criterio difusos. En Puerta J.M., Gámez J.A., Dorronsoro B., Barrenechea E., Troncoso A., Baruque B. \& Galar M. (Ed.), Actas de la XVI Conferencia CAEPIA, 459-469. Albacete, España.

Costa, N.R. \& Lourenço, J. (2016). Multiresponse problems: Desirability and other optimization approaches. Journal of Chemometrics, 30(12), 702-714. https://doi.org/10.1002/cem.2848

Da Silva, I.N., Spatti, D.H., Flauzino-Andrade, R., Bartocci-Liboni, L.H. \& dos Reis-Alves, S.F. (2017). Artificial Neural Networks. Springer.

Derringer, G.C. (1994). A balancing act: Optimizing a products properties. Quality Progress, 27(6), 51-58.

Derringer, G. \& Suich, R. (1980). Simultaneous optimization of several response variables. Journal of Quality Technology, 12(4), 214-219. https://doi.org/10.1080/00224065.1980.11980968

Fritsch, S., Guenther, F. \& Wright, M.N. (2019). Neuralnet: Training of Neural Networks. R package version 1.44.2.

Genz, A., Bretz, F., Miwa, T., Mi, X., Leisch, F., Scheipl, F. \& Hothorn, T. (2019). Mvtnorm: Multivariate Normal and t Distributions. R package version 1.0-11.

Gutiérrez-Pulido, H. \& De la Vara-Salazar, R. (2012). Análisis y diseño de experimentos. (Tercera ed.). México: McGraw-Hill Interamericana Editores, S.A. de C.V.

Harrington, E.C. (1965). The desirability function. Industrial Quality Control, 21, 494-498.

Hwang, C.-L. \& Yoon, K. (1981). Multiple attribute decision making. Berlin Heidelberg: Springer-Verlag.

Ic, Y. \& Yildirim, S. (2012). Improvement of a product design using multi criteria decision making methods with taguchi method. Journal of the Faculty of Engineering and Architecture of Gazi University, 27, 447-458.

Ic, Y. \& Yildirim, S. (2013). Moora-based taguchi optimisation for improving product or process quality. International Journal of Production Research, 51(11), 3321-3341. https://doi.org/10.1080/ 00207543.2013.774471

Khuri, A.I. (1981). Simultaneous optimization of multiple responses represented by polynomial. Technometrics, 23(4), 363-375.

Khuri, A.I. (2017). Response surface methodology and its applications in agricultural and food science. Biometrics \& Biostatistics International Journal, 5(5), 2-11. https://doi.org/10.15406/bbij.2017.05.00141

Kilickap, E., Yardimeden, A. \& Çelik, Y.H. (2017). Mathematical modelling and optimization of cutting force, tool wear and surface roughness by using artificial neural network and response surface methodology in milling of ti-6242s. Applied Sciences, 7(10), 1064. https://www.researchgate.net/deref/ http\%3A\%2F\%2Fdx.doi.org\%2F10.3390\%2Fapp7101064

Kim, Z., Shin, Y., Yu, J., Kim, G. \& Hwang, S. (2019). Development of NOx removal process for LNG evaporation system: Comparative assessment between response surface methodology (RSM) and artificial neural network (ANN). Journal of Industrial and Engineering Chemistry, 74, 136-147. 
Kuhn, M. (2016). desirability: Function Optimization and Ranking via Desirability Functions. R package version 2.1.

Kuo, Y., Yang, T., \& Huang, G.-W. (2008). The use of a grey-based taguchi method for optimizing multi-response simulation problems. Engineering Optimization, 40, 517-528. https://doi. org $/ 10.1080 / 03052150701857645$

Lu, J., Feng, X., Han, Y. \& Xue, C. (2013). Optimisation of subcritical fluid extraction of carotenoids and chlorophyll a from laminaria japonica aresh by response surface methodology. Journal of the Science of Food and Agriculture, 94, 139-145. https:// doi.org/10.1002/jsfa.6224

Majid, B., Khanmohammadi, O., Morteza, Y. \& Joshua, I. (2012). A state-of the-art survey of topsis applications. Expert Systems with Applications, 39(17), 13051-13069. https://www.researchgate.net/deref/http $\% 3 \mathrm{~A} \% 2 \mathrm{~F} \% 2 \mathrm{Fdx}$.doi.org\%2F10.1016\%2Fj. eswa.2012.05.056

Manohar, M., Joseph, J., Selvaraj, T. \& Sivakumar, D. (2013). Application of box behnken design to optimize the parameters for turning inconel 718 using coated carbide tools. International Journal of Scientific \& Engineering Research, 4(4), 620-644.

Maran, J.P. \& Priya, B. (2015). Ultrasonics Sonochemistry, 23, 192200.

Miettinen, K. (2008). Introduction to multiobjective optimization: Noninteractive approaches. En Branke J., Deb K., Miettinen K. \& Słowiński R. (Ed.), Multiobjective Optimization. Lecture Notes in Computer Science (pp. 1-26). Berlin Heidelberg: Springer.

Najafi, B., Faizollahzadeh-Ardabili, S., Mosavi, A., Shamshirband, S. \& Rabczuk, T. (2018). An intelligent artificial neural network-response surface methodology method for accessing the optimum biodiesel and diesel fuel blending conditions in a diesel engine from the viewpoint of exergy and energy analysis. Energies, 11(4), 860.

Phadke, M.S. (1989). Quality engineering using robust design. Englewood Cliffs, NJ: PTR Prentice-Hall Inc.
R Core Team. (2019). R: A Language and environment for statistical computing. Vienna, Austria: R Foundation for Statistical Computing.

Rocha, L.C., de Paiva, A.P., Paiva, E.J. \& Balestrassi, P.P. (2015). Comparing dea and principal component analysis in the multiobjective optimization of p-gmaw process. Journal of the Brazilian Society of Mechanical Sciences and Engineering, 38, 2513-2526. http://dx.doi.org/10.1007\%2Fs40430-015-0355-Z

Shafi, J., Sun, Z., Ji, M., Gu, Z. \& Ahmad, W. (2018). ANN and RSM based modelling for optimization of cell dry mass of Bacillus sp. strain B67 and its antifungal activity against Botrytis cinerea. Biotechnology \& Biotechnological Equipment, 2(1), 58-68. https://doi.org/10.1080/13102818.2017.1379359

Šibalija, T.V. \& Majstorović, V.D. (2016). Advanced multiresponse process optimisation: An intelligent and integrated approach. Switzerland: Springer International Publishing.

Sivakumar, T., Manavalan, R., Muralidharan, C. \& Valliappan, K. (2007). Multi-criteria decision making approach and experimental design as chemometric tools to optimize HPLC Separation of Domperidone and Pantoprazole. Journal of Pharmaceutical and Biomedical Analysis, 43(5), 1842-1848. https://doi.org/10.1016/j. jpba.2006.12.007

Tong, L.-I. \& Hsieh, K.-L. (2001). A novel means of applying neural networks to optimize the multiresponse problem. Quality Engineering, 13, 11-18. https://doi.org/10.1080/08982110108918619

Venables, W.N. \& Ripley, B.D. (2016). nnet: Feed-Forward Neural Networks and Multinomial Log-Linear Models. R package version 7.3-12.

Yang, T. \& Chou, P. (2005). Solving a multiresponse simulationoptimization problem with discrete variables using a multiple- attribute decision-making method. Mathematics and Computers in Simulation, 68, 9-21. https://doi.org/10.1016/j. matcom.2004.09.004 